\title{
Comments on Misconceptions About the Management of Low Back Pain: A Brief Note for Physiotherapists by Musa Sani Danazumi
}

\author{
Faizan Zaffar Kashoo (iD) ${ }^{1, *}$ and Mehrunnisha Ahmad (iD) ${ }^{2}$ \\ ${ }^{1}$ Department of Physical Therapy and Health Rehabilitation, College of Applied Medical Sciences, Majmaah University, Majmaah, Saudi Arabia \\ ${ }^{2}$ Department of Nursing, College of Applied Medical Sciences, Majmaah University, Majmaah, Saudi Arabia \\ "Corresponding author: Department of Physical Therapy and Health Rehabilitation, College of Applied Medical Sciences, Majmaah University, Majmaah, Saudi Arabia. Tel: \\ +966-538149226, Email: f.kashoo@mu.edu.sa
}

Received 2020 March 29; Accepted 2020 April 24.

Keywords: Letter to the Editor, Critical Appraisal, Low Back Pain

\section{Dear Editor,}

We read the review article with interest titled "Misconceptions about the Management of Low Back Pain: A Brief Note for Physiotherapists by Musa Sani Danazumi" (1). The article is about physical therapy management in low back pain. We would like to congratulate the authors on this well-written review. However, there are few concerns related to the scope of management of low back pain by a physical therapist.

First, the author commented on the lack of robust scientific evidence and physical therapy techniques used for back pain. We differ from the author's conceptualization of current evidence-based practices and pretend that the author might have missed the recent systematic review and meta-analysis about the effectiveness of techniques such as Maitland, core muscle strengthening, McKenzie exercises for back pain (2). Moreover, to meet therapeutic goals of the treatment, physiotherapists barely use a single technique, as conditions such as backache contain multiple symptoms that each should be addressed with different techniques. However, it does not mean, if administered in isolation, would not be effective (3).

Second, the medical imaging technique is of paramount importance in cases with severe diseases, such as sacral tumors and other serious medical conditions that need immediate surgical interventions (4). Moreover, the imaging prescription by a physical therapist is limited, as a recent study reported the vast discrepancy between the symptoms perceived by the patient and the extent of damage shown in the radiograph. According to this new age evidence-based practice, physical therapists abstain from prescribing unnecessary imaging, as there are validated tools that would provide a better clinical picture of the patient (5).

Third, related to the prescription of surgery, we entirely agree with the author that if the neurological complications are progressive, the surgery is necessary. Surgery is the last option to address neural compression (6). The pre- and post-operative training are essential to regain the normal functional ability. The physical therapist plays a vital role in improving the general health status of those who had surgical interventions. The pre-operative training includes breathing exercises, strengthening of venerable muscles, and consultations related to the pain. However, there is no drug to prevent the effects of immobilization and muscle weakness. The post-operative training ensures that the patient regains complete functional independence.

Fourth, the author reported a narrow scope of thought; however, the write-up is not related to the title of the paragraph. A physiotherapist should use an arsenal of therapeutic techniques, because patients with back pain have a wide range of clinical presentations (7). Applying a unified technique for all cases is not appropriate. Therefore a tailor-made approach to meet the patient's functional needs and the therapeutic goals is the best way to justify the intervention provided to the patients.

In conclusion, The review article provides useful but limited information about the therapeutic effectiveness and the validated tools used in backache treatment by the physical therapist.

\section{Footnotes}

Authors' Contribution: Study concept and design: FK and MA. Analysis and interpretation of data: FK and MA. 
Drafting of the manuscript: FK. Critical revision of the manuscript for important intellectual content: FK and MA. Conflict of Interests: Authors declare no conflict of interest.

\section{Funding/Support: None.}

\section{References}

1. Danazumi MS. Misconceptions about the management of low back pain: A brief note for physiotherapists. Middle East J Rehabil Health Stud. 2020;7(2). doi:10.5812/mejrh.101154.

2. Pires D, Cruz EB, Gomes LA, Nunes C. How do physical therapists measure treatment outcomes in adults with chronic low back pain? A systematic review. Phys Ther. 2020. doi:10.1093/ptj/pzaa030. [PubMed: 32115634].
3. Kashoo FZ, Ahmad M. Effect of sensory integration on attention span among children with infantile hemiplegia. Int J Health Sci (Qassim). 2019;13(3):29-33. [PubMed: 31123437]. [PubMed Central: PMC6512152].

4. Lemmers GPG, van Lankveld W, Westert GP, van der Wees PJ, Staal JB. Imaging versus no imaging for low back pain: A systematic review, measuring costs, healthcare utilization and absence from work. Eur Spine J. 2019;28(5):937-50. doi: 10.1007/s00586-019-05918-1. [PubMed: 30796513].

5. Alqahtani MM, Kashoo FZ. Physical therapy in cervicogenic dizziness. Saudi J Heal Sci. 2020;9(1):1-6.

6. Rafique MK, Taqi A. Review article-The causes, prevention and management of post spinal backache: An overview. Anaesth Pain Intens Care. 2019.

7. Kashoo F, Ahmad M. Challenge is the key to stroke rehabilitation. Biomed Res. 2018;29(21). doi: 10.4066/biomedicalresearch.29-18-1076. 